\title{
Bone regeneration potential of human dental pulp stem cells derived from elderly patients and induced by a helioxanthin derivative
}

\section{Marika Sato}

Department of Oral and Maxillofacial surgery, Tokyo Medical University

YOKO KAWASE-KOGA ( $\nabla$ kogay@tokyo-med.ac.jp )

Tokyo Ika Daigaku

Daiki Yamakawa

Department of Oral and Maxillofacial Surgery, Tokyo Medical University

Yasuyuki Fujii

Department of Oral and Maxillofacial Surgery, Tokyo Medical University

\section{Daichi Chikazu}

Department of Oral and Maxillofacial Surgery, Tokyo Medical University

\section{Research}

Keywords: Dental pulp stem cell, Bone regeneration, Cell-sheet technology, Age, Osteogenic differentiation

Posted Date: March 5th, 2020

DOI: https://doi.org/10.21203/rs.3.rs-16130/v1

License: (9) This work is licensed under a Creative Commons Attribution 4.0 International License. Read Full License 


\section{Abstract}

Background: Human dental pulp stem cells (DPSCs) have the ability to differentiate into multiple lineage cell types including adipogenic, neurogenic, and osteogenic cells. Dental pulp stem cells can be easily collected from extracted teeth and are now considered to be a type of mesenchymal stem cell with higher clonogenic and proliferative potential than bone marrow stem cells. Previous studies have described the osteogenic ability of DPSCs that were isolated from only young patients. However, in fact, elderly patients more frequently require bone regenerative therapy-for example, in alveolar bone defects resulting from periodontal disease-than young patients. We previously reported that 4-(4methoxyphenyl)pyrido[40,30:4,5]thieno[2,3-b]pyridine-2-carboxamide (TH), a helioxanthin derivative, induces osteogenic differentiation of DPSCs derived from young patients. However, the effect of TH on the osteogenic differentiation potential of DPSCs derived from elderly patients remains unknown. Therefore, the present study aimed to examine and compare the osteogenic differentiation potential of TH-induced DPSCs from elderly patients and young patients to explore the potential clinical use of DPSCs for elderly patients.

Methods: Dental pulp stem cells were obtained from the dental pulp of teeth of healthy young patients (18-39 years old) and healthy elderly patients (40-67 years old) and cultured in regular medium and osteogenic medium with or without $\mathrm{TH}$. We assessed the morphological characteristics, proliferation, and osteogenic differentiation of DPSCs from both young and elderly patients. Moreover, DPSC sheets were transplanted into mouse calvarial defects to investigate osteogenesis of TH-induced DPSCs in vivo.

Results: We demonstrated that osteogenic conditions with TH induce the osteogenic differentiation and osteogenesis of DPSCs from elderly patients as well as young patients more than osteogenic conditions without TH.

Conclusions: Our results suggested that the osteogenic differentiation potential of DPSCs from elderly patients is as high as that of DPSCs from young patients. Moreover, TH-induced DPSCs show increased osteogenic differentiation potential, and they can be a useful cell source for bone regenerative therapy for not only young patients but also elderly patients.

\section{Background}

Bone regenerative therapy is in great demand nowadays for alveolar bone loss caused by periodontitis, tumor resection, traumatic injury, and dental implant therapy. In the clinical field, bone regenerative therapy is required especially for elderly patients over 40 years of age owing to the high morbidity rate of periodontitis. Mesenchymal stem cells (MSCs) derived from bone marrow (BMSCs) and adipose tissue are known to be a useful cell source for tissue engineering. However, collecting BMSCs is challenging owing to the necessity of using an invasive technique. To date, bone augmentation techniques such as bone harvesting from the iliac crest under general anesthesia, autogenous bone grafting from the mandible, and artificial bone grafting have been used for alveolar bone defect in periodontitis treatment 
and dental implant treatment. However, these present a high likelihood of infection and unexpected resorption.

Human dental pulp stem cells (DPSCs) were identified in 2000, and they have a high potential to proliferate, self-renew, and differentiate into multiple lineage cells such as adipogenic, neurogenic, and osteogenic cells [1-3]. The clonogenic and proliferative potential of DPSCs is higher than that of BMSCs. Moreover, DPSCs can be obtained from extracted teeth that are usually discarded, and the method of collecting DPSCs is non-invasive and uncomplicated without ethical issues. Thus, DPSCs have the potential to be a promising cell source for regenerative therapy.

Several studies report that the number of MSCs and stem cell functions such as the ability to self-renew decline with increasing age [4]. Furthermore, the proliferation and differentiation potential of MSCs also declines with age and after repeated passage [5-8], owing to telomere shortening, DNA damage, and epigenetic changes in transcriptional regulation [9]. Although many studies have investigated the differentiation potential of DPSCs isolated from young patients, little information is available about osteogenic differentiation potential of DPSCs derived from elderly patients. In fact, the volume of dental pulp tissue decreases with age owing to secondary dentin formation and root canal mineralization [10]. Thus, a simple and effective method of transplantation is required to overcome these limitations.

In our previous study, we showed that 4-(4-methoxyphenyl)pyrido[40,30:4,5]thieno[2,3-b]pyridine-2carboxamide (TH)-a small-molecule derivative of helioxanthin-induces osteogenic differentiation of DPSCs isolated from young patients in vitro and in vivo, using transplantation of DPSCs with cell-sheet technology. The cell-sheet technology facilitates stem cell harvest and transplantation without the need for digestive enzymes and scaffolds, resulting in minimal cell loss and damage and avoiding inflammatory responses caused by biodegradable scaffolds [11]. However, the effect of TH on the osteogenic differentiation potential of DPSCs derived from elderly patients remains unknown.

In the present study, we investigated and compared the osteogenic differentiation potential and osteogenesis of TH-induced DPSCs from elderly and young patients in vitro and in vivo to explore the potential clinical use of DPSCs.

\section{Methods}

\section{Isolation of dental pulp stem cells}

Human dental pulp stem cells were isolated from extracted teeth from healthy young (16-39-year-old) and elderly (40-76-year-old) patients (hereafter referred to as "young DPSCs" and "elderly DPSCs", respectively)-after obtaining informed consents-at Tokyo Medical University Hospital. This study was approved by the institutional ethics committee of the Faculty of Medicine, Tokyo Medical University, Japan (approval no. 3486). Dental pulp was extracted and minced into small pieces, followed by enzymatic digestion using $3 \mathrm{mg} / \mathrm{mL}$ collagenase type I (Sigma-Aldrich) for $45 \mathrm{~min}$ at $37^{\circ} \mathrm{C}$; single-cell suspensions were obtained by passing the cells through a $70 \mu \mathrm{m}$ cell strainer. The isolated cells were 
plated at a density of $1 \times 10^{5}$ cells on $100 \mathrm{~mm}$ dishes and cultured in alpha-modified Eagle's medium (aMEM; Gibco/BRL) supplemented with $15 \%$ fetal bovine serum (FBS; Biowest) and $1 \%$ penicillin/streptomycin (P/S; Wako Pure Chemical Industries). Cells were passaged at $70 \%$ confluence. Young and elderly DPSCs in passage 3 (P3) or P4 were used for experiments. Dental pulp stem cells were cultured in 12-well plates containing osteogenic differentiation media with and without TH. We used aMEM supplemented with $10 \% \mathrm{FBS}, 1 \% \mathrm{P} / \mathrm{S}$, and $10 \mathrm{nM}$ dexamethasone (Wako Pure Chemical Industries) for osteogenic induction (osteogenic medium [OM]). We added TH (Takeda Chemical Industries) at the optimal concentration of $10^{-6} \mathrm{M}$ to the $\mathrm{OM}$, as described previously [12].

\section{Cell Proliferation}

Dental pulp stem cells $\left(1 \times 10^{5}\right.$ cells $)$ were cultured in $100 \mathrm{~mm}$ dishes at a density of $1 \times 10^{5}$ cells until they reached $70 \%$ confluence. To calculate the initial percentage of DPSCs from young and elderly patients, the number of cells initially plated and the number of cells after detachment at $70 \%$ confluence were counted. Cell proliferation of young and elderly DPSCs was compared by collecting and counting cells at P1, P2, and P3.

\section{Flow cytometry analysis}

A single-cell suspension was obtained by detaching the cells using $0.25 \%$ trypsin and washing them with phosphate-buffered saline (PBS). Cells were fixed with $10 \% \mathrm{FBS}$ for $10 \mathrm{~min}$ at $37^{\circ} \mathrm{C}$ and then incubated with the following antibodies for $90 \mathrm{~min}$ at $4{ }^{\circ} \mathrm{C}$ : fluorescein isothiocyanate (FITC)-conjugated antihuman CD14, phycoerythrin (PE)-conjugated human CD29, FITC-conjugated human CD34, FITCconjugated human CD44, PE- conjugated human CD73, FITC-conjugated human CD81, PE- conjugated human CD90, FITC-conjugated human CD105, and FITC-conjugated human CD146 (BioLegend). Cells were washed with PBS and then fixed in $4 \%$ paraformaldehyde (PFA) for 10 min at $4{ }^{\circ} \mathrm{C}$. Non-labelled cells were used as negative controls. Cells were analyzed using a flow cytometer (BD Biosciences), and data analysis was performed using FlowJo software (FlowJo; FlowJo, LLC).

\section{Alizarin red S staining}

Young and elderly DPSCs were cultured in OM with or without TH for 14 days and stained with alizarin red S (Sigma-Aldrich), as described previously. In brief, cells were fixed with $10 \%$ formaldehyde in PBS for 10 min at $4{ }^{\circ} \mathrm{C}$, followed by two washes with distilled water. Next, the cells were stained in $1 \%$ alizarin red $\mathrm{S}$ solution for $15 \mathrm{~min}$. After staining, they were washed twice with distilled water.

\section{Alkaline phosphatase staining}

Young and elderly DPSCs were stained with alkaline phosphatase (ALP) as described previously. In brief, the cells were fixed in $70 \%$ ethanol after they were rinsed with PBS, followed by staining for 10 min with $0.01 \%$ naphthol AS-MX phosphate (Sigma-Aldrich), using $1 \%$ N,N-dimethyl formamide (Wako Pure Chemical Industries) as the substrate and $0.06 \%$ Fast BB salt (Sigma-Aldrich) as a coupler. 


\section{Reverse transcription-polymerase chain reaction analysis}

Total RNA from young and elderly DPSCs cultured in regular medium (RM Dulbecco's Modified Eagle Medium [DMEM, GIBCO/BRL] supplemented with $10 \%$ FBS and $1 \% \mathrm{P} / \mathrm{S}$ ), OM, and OM with TH was isolated using TRIzol (Invitrogen), and reverse transcription was performed using QuantiTect Reverse Transcription kit (Qiagen) according to the manufacturer's instructions. Real-time polymerase chain reaction of Runx2, an osteoprogenitor maker, Alp and Colla1, early markers of osteoblast differentiation, and osteocalcin, a mature osteoblast marker, was performed in a Lightcycler 96 (Roche Diagnostics) using THUNDERBIRD SYBR qPCR Mix (Toyobo), as described previously. GAPDH was used as an endogenous control. The primer sequences used in this study are presented in Table 1.

\section{Transplantation of young and elderly dental pulp stem cell sheets into a mouse calvarial defect model}

A mouse calvarial defect model was used for animal experiments, as described previously, following the guidelines of Animal Care and Use committee of the Faculty of Medicine, Tokyo Medical University. In brief, young and elderly DPSC sheets were cultured on temperature-responsive dishes in OM with TH treatment for 14 days, and each sheet was transplanted into the calvarial defects ( $3.5 \mathrm{~mm}$ in diameter) created in the right parietal bone using biopsy punches (Kai Corporation). Four mice were used in each experimental group. Eight weeks after transplantation, mice were sacrificed, and calvaria were harvested.

\section{Radiological evaluation}

The calvarial defects were examined by micro-computed tomography (micro-CT; SMX-90CT; Shimadzu) The scanning conditions were as follows: $90 \mathrm{kV}, 110 \mu \mathrm{A}$, and a field of view (XY) of $10 \mathrm{~mm}$; the resolution of one CT slice was $512 \times 512$ pixels. The data were reconstructed and analyzed using morphometric software TRI/3D-BON (Ratoc System Engineering). Bone volume (BV), bone mineral content (BMC), and bone mineral density (BMD) of a $5 \times 5 \times 3 \mathrm{~mm}^{3}$ cuboid area located in the center of the initial defect area were calculated.

\section{Statistical analysis}

Each experiment was replicated at least three times. Statistical analysis was performed using SPSS 24.0 software (IBM); all data are expressed as mean \pm SD. Statistical significance was evaluated using oneway ANOVA, and $p$ values were calculated with Student $t$ test. $\mathrm{P}<0.05$ or $\mathrm{P}<0.01$ was considered to indicate statistically significant differences between two groups.

\section{Results}

\section{Characterization of young and elderly dental pulp stem cells}

At initial culture, young and elderly DPSCs showed morphologically similar spindle shape (Fig. 1A). The average number of DPSCs derived from young and elderly patients was $70 \%$ and $42 \%$ of the total number of cells collected, respectively (Table 2). Proliferation potential of elderly DPSCs in P1 was 
significantly lower than that of young DPSCs (Fig. 1B). However, in P2, the proliferation potential of elderly DPSCs increased and was not significantly different from that of young DPSCs, it remained at a high level in P3 (Fig. 1B). Flow cytometry analysis indicated that young and elderly DPSCs showed a similar pattern of expression of MSC surface markers, being positive for CD29, CD44, CD73, CD81, CD90, and CD105 and negative for CD14 and CD34 (Fig. 2), which are the minimal criteria for DPSCs.

\section{Effect of TH on osteogenic differentiation of young and elderly dental pulp stem cells}

To determine the effect of TH on elderly DPSCs, we compared the osteogenic differentiation potential of TH-induced DPSCs from young and elderly patients. Alizarin red S staining showed that in both young and elderly DPSCs, higher calcification was observed in DPSCs cultured in OM with TH than in OM without TH (Fig. 3A). Moreover, ALP staining showed that young and elderly TH-induced DPSCs displayed stronger ALP activity than DPSCs in OM (Fig. 3B). Real-time PCR showed that TH upregulated osteogenic differentiation markers in DPSCs derived from both young and elderly patients, although the difference was not significant. In addition, in DPSCs from both young and elderly patients, Colla 1, Alp, osteocalcin, and Runx2 were found to be more highly expressed in TH-induced DPSCs than in DPSCs cultured in OM, although the difference was not statistically significant (Fig. 3C).

\section{In vivo osteogenesis of TH-induced young and elderly dental pulp stem cells}

To determine the osteogenesis of TH-induced elderly DPSCs in vivo, we cultured young and elderly DPSCs on temperature-responsive dishes (12-well, $3.5 \mathrm{~cm}^{2} /$ well) under osteogenic conditions with TH for 2 weeks, then harvested the DPSC sheets, and transplanted them into the calvarial defects of mice. Eight weeks after transplantation, micro-CT images were taken to compare the bone regeneration potential of young DPSCS with that of elderly DPSCS (Fig. 4A). The micro-CT images revealed that the newly formed bone was detected at the edge of the defect in the elderly DPSC group as well as in the young DPSC group, whereas no bone regeneration was observed in the control group (Fig. 4B). Quantitative evaluation indicated no significant differences in BV and BMC between young and elderly DPSC groups; however, BV and BMC in the elderly DPSC group were significantly higher than those in the control group (Fig. 4C).

\section{Discussion}

In the present study, we studied the osteogenic differentiation potential of TH-induced DPSCs from elderly patients and compared it with that of DPSCs from young patients. In comparison with BMSCs [2] and adipose tissue-derived stem cells (ADSCs) [13], DPSCs have a higher proliferative potential and ease of accessibility, and the method to harvest them is less invasive; therefore, DPSCs can be a promising cell source for regenerative medicine. The MSCs and ADSCs show an age-dependent decline in their proliferative ability and regenerating capacity $[4,14,15]$. In fact, the volume of pulp tissue decreases with age owing to secondary dentin formation and root canal mineralization [10]. The present study demonstrated that the number of collected cells from the pulp tissue of elderly patients was lower than that collected from young patients, which agrees with previously published data [16]. Both the quality and 
quantity of DPSCs are important in regenerative therapy; therefore, these age-related changes can make the clinical use of DPSCs from elderly patients challenging. However, the effect of age in bone regeneration has not yet been clear. Thus, age-independent culture methods that render the DPSCs to maintain a high proliferation and differentiation potential are desirable in tissue engineering therapy. Although some studies have reported an efficient system that induces osteogenic differentiation of DPSCs, such as producing the scaffold materials [17], and modifying the medium[18], its effect in aging patients has not yet been reported. We previously demonstrated that $\mathrm{TH}$, a small osteogenic molecule, induced osteogenic differentiation of DPSCs from young patients in vivo and in vitro [12]. With this method, it is possible to culture DPSCs with a high proliferation and differentiation potential. In the present study, to assess whether TH can be used in DPSCs derived from elderly patients, the effect of TH on the osteogenic differentiation potential of DPSCs from elderly patients was examined in a mouse calvarial defect model and compared with that of DPSCs from young patients.

It has been shown that MSCs from elderly patients have a lower proliferative potential even after the initial passage $[6,19]$. In the present study, we demonstrated that, at initial culture and P1, the proliferation potential of DPSCs from elderly patients was significantly lower than that of DPSCs from young patients. In contrast, no significant differences were found between the proliferative ability of elderly DPSCs and young DPSCs after P2, and the proliferative ability of elderly DPSCs was maintained at a high level even at P3, indicating that once the pulp cells gain "stemness" after passaging, the high proliferation potential of DPSCs is independent of age even though the initial number of collected cells is low in elderly patients. Similar results were obtained in a previous study in which they observed a high proliferative ability of DPSCs derived from different age groups including elderly patients, and the ability was also maintained at P2 in all age groups [20].

We previously reported that the optimal concentration of $\mathrm{TH}$ that is the most effective on osteogenic differentiation of DPSCs is approximately $10^{-6} \mathrm{M}$ and that OM is necessary for osteogenic induction when DPSCs are cultured with TH $[12,21]$. In the present study, we used the same concentration of TH and the same culture method for both young and elderly DPSCs. Our data demonstrated that compared with $\mathrm{OM}$ alone, $\mathrm{OM}$ with $\mathrm{TH}$ increased-although not significantly-the expression levels of Colla 1, Alp, osteocalcin, and Runx2 in both young and elderly DPSCs. Moreover, ALP staining and alizarin red S staining showed that TH induced higher ALP activity and more mineral deposition in both young and elderly groups, indicating that $\mathrm{TH}$ is effective in inducing osteogenic differentiation of not only young DPSCs but also elderly DPSCs.

As we previously reported, the cell sheet method using TH-induced DPSCs enables easier and safer transplantation of DPSCs [12]. Bone regenerative therapy using transplantation of DPSC sheets in combination with $\mathrm{TH}$ requires no scaffolds or growth factors, thus overcoming challenges associated with invasive autologous bone harvesting and ethical issues associated with allogenic bone transplantation and recombinant protein use. Moreover, previous studies have shown that DPSCs have a higher immunosuppressive activity and ability to prevent T-cell alloreactivity than BMSCs [22, 23], which can be advantageous for the clinical use of DPSC sheets. Furthermore, this finding was supported by the 
subsequent in vivo study using a mouse calvarial defect model. We evaluated the osteogenic potential of TH-induced DPSCs from elderly patients using a mouse calvarial defect model, which closely resembled clinical settings. Notably, transplants with elderly DPSCs and young DPSCs showed similar new bone formation at the edge of the calvarial defect. Moreover, BV and BMC in the transplants with elderly DPSCs were considerably higher than those in control, but no significant difference was found between transplants with elderly and young DPSCs, indicating that elderly DPSCs treated with TH have a high potential for osteogenic differentiation and osteogenesis, which is not inferior to that of young DPSCs. Previous studies have shown that using scaffolds increased the osteogenesis of DPSCs $[24,25]$ derived from young patients. However, TH improved the osteogenesis of DPSCs without any scaffolds and independent of the age of the source tissue. In conclusion, these radiological findings confirm the advantage of using $\mathrm{TH}$ for bone regenerative therapy to the patients irrespective of patient age.

\section{Conclusion}

Our study demonstrated that TH is effective in inducing osteogenic differentiation of DPSCs from elderly patients. Moreover, TH-induced DPSC from elderly patients have a high potential for osteogenesis as well as young patients in vivo, indicating that TH-induced DPSCs from elderly patients can be a useful cell source for bone regenerative medicine.

\section{Declarations}

\section{Acknowledgements}

We thank members of the Ohba-Chung laboratory for their helpful input.

\section{Funding}

This work was funded by a Tokyo Medical University Research Grant, a Grants-in-Aid of the Ishidsu Shun Memorial Scholarship, and a Grant-in-Aid for Scientific Research C (Nos. 25670852, 16K11700) from the Ministry of Education, Culture, Sports, Science and Technology, Japan.

\section{Availability of data and materials}

All data generated or analyzed for this study are included in this published article.

\section{Author's contributions}

YK, MS, and DC conceived and designed the experiments. MS, DY, and YF were responsible for sample collection. MS and YK performed the experiments and wrote the manuscript. MS and YK analyzed the data. All authors discussed the results and commented on the manuscript. All authors read and approved the final manuscript.

\section{Ethics approval}


This study was performed after receiving written consent from all patients and was approved by the institutional ethics committee of the Faculty of Medicine, Tokyo Medical University (approval no.3486). Animal experiments were performed according to a protocol approved by the Animal Care and Use committee of the Faculty of Medicine, Tokyo Medical University.

\section{Consent for publication}

Not applicable.

\section{Competing interests}

There are no conflicts of interest associated with this study.

\section{Abbreviations}

ADSCs: adipose tissue-derived stem cells

ALP: alkaline phosphatase

BMC: bone mineral content

BMD: bone mineral density

BMP: bone morphogenic protein

BMSCs: bone marrow stem cells

BV: bone volume

COLIA1: Type I collagen alpha 1

DPSCs: human dental pulp stem cells

FITC: fluorescein isothiocyanate

MSCs: mesenchymal stem cells

OM: osteogenic medium

P/S: penicillin/streptomycin

PBS: phosphate-buffered saline

PE: phycoerythrin

RM: regular medium 
SD: standard deviation

TH: 4-(4-methoxyphenyl) pyrido[40,30:4,5]thieno[2,3-b]pyridine-2-carboxamide

aMEM: alpha-modified Eagle's medium

\section{References}

1. Gronthos S, Brahim J, Li W, Fisher LW, Cherman N, Boyde A, et al. Stem cell properties of human dental pulp stem cells. J Dent Res. 2002;81:531-5.

2. Gronthos S, Mankani M, Brahim J, Robey PG, Shi S. Postnatal human dental pulp stem cells (DPSCs) in vitro and in vivo. Proc Natl Acad Sci USA. 2000;97:13625-30.

3. Tamaki $Y$, Nakahara T, Ishikawa $H$, Sato $S$. In vitro analysis of mesenchymal stem cells derived from human teeth and bone marrow. Odontology. 2013;101:121-32.

4. Liu L, Rando TA. Manifestations and mechanisms of stem cell aging. J Cell Biol. 2011;193:257-66.

5. Stolzing A, Jones E, McGonagle D, Scutt A. Age-related changes in human bone marrow-derived mesenchymal stem cells: consequences for cell therapies. Mech Ageing Dev. 2008;129:163-73.

6. Alt EU, Senst C, Murthy SN, Slakey DP, Dupin CL, Chaffin AE, et al. Aging alters tissue resident mesenchymal stem cell properties. Stem Cell Res. 2012;8:215-25.

7. Wu W, Niklason L, Steinbacher DM. The effect of age on human adipose-derived stem cells. Plast Reconstr Surg. 2013;131:27-37.

8. Stenderup K, Justesen J, Clausen C, Kassem M. Aging is associated with decreased maximal life span and accelerated senescence of bone marrow stromal cells. Bone. 2003;33:919-26.

9. Smith JA, Daniel R. Stem cells and aging: a chicken-or-the-egg issue? Aging Dis. 2012;3:260-8.

10. Murray PE, Stanley HR, Matthews JB, Sloan AJ, Smith AJ. Age-related odontometric changes of human teeth. Oral Surg Oral Med Oral Pathol Oral Radiol Endod. 2002;93:474-82.

11. Wei F, Qu C, Song T, Ding G, Fan Z, Liu D, et al. Vitamin C treatment promotes mesenchymal stem cell sheet formation and tissue regeneration by elevating telomerase activity. J Cell Physiol. 2012;227:3216-24.

12. Fujii Y, Kawase-Koga Y, Hojo H, Yano F, Sato M, Chung Ul, et al. Bone regeneration by human dental pulp stem cells using a helioxanthin derivative and cell-sheet technology. Stem Cell Res Ther. 2018;9:24.

13. Stanko P, Kaiserova K, Altanerova V, Altaner C. Comparison of human mesenchymal stem cells derived from dental pulp, bone marrow, adipose tissue, and umbilical cord tissue by gene expression. Biomed Pap Med Fac Univ Palacky Olomouc Czech Repub. 2014;158:373-7.

14. Siegel G,Kluba T, Hermanutz-Klein U, Bieback K, Northoff H, Schäfer R. Phenotype, donor age and gender affect function of human bone marrow-derived mesenchymal stromal cells. BMC Medicine. 2013;11:146. 
15. Alraies A, Alaidaroos NY, Waddington RJ, Moseley R, Sloan AJ. Variation in human dental pulp stem cell ageing profiles reflect contrasting proliferative and regenerative capabilities. BMC Cell Biol. 2017;18:12.

16. lida K, Takeda-Kawaguchi T, Tezuka Y, Kunisada T, Shibata T, Tezuka K. Hypoxia enhances colony formation and proliferation but inhibits differentiation of human dental pulp cells. Arch Oral Biol. 2010;55:648-54.

17. Amir LR, Suniarti DF, Utami S, Abbas B. Chitosan as a potential osteogenic factor compared with dexamethasone in cultured macaque dental pulp stromal cells.Cell Tissue Res. 2014;358:407-15.

18. Wu J,Li N, Fan Y, Wang Y, Gu Y, Li Z, et al. The conditioned medium of calcined tooth powder promotes the osteogenic and odontogenic differentiation of human dental pulp stem cells via MAPK signaling pathways. Stem Cells Int. 2019;4793518:13.

19. Baxter MA, Wynn RF, Jowitt SN, Wraith JE, Fairbairn LJ, Bellantuono I. Study of telomere length reveals rapid aging of human marrow stromal cells following in vitro expansion. Stem Cells. 2004;22:675-82.

20. Bressan E, Ferroni L, Gardin C, Pinton P, Stellini E, Botticelli D, et al. Donor age-related biologicalproperties of human dental pulp stem cells change in nanostructured scaffolds. PLoS One. 2012;7:e49146.

21. Ohba S, Nakajima K, Komiyama Y, Kugimiya F, Igawa $K$, Itaka $K$, et al. A novel osteogenic helioxanthin-derivative acts in a BMP-dependent manner.Biochem Biophys Res Commun. 2007;357:854-60.

22. Pierdomenico L, Bonsi L, Calvitti M, Rondelli D, Arpinati M, Chirumbolo G, et al. Multipotent mesenchymal stem cells with immunosuppressive activity can be easily isolated from dental pulp.Transplantation. 2005;80:836-42.

23. Yamada Y, Ito K, Nakamura S, Ueda M, Nagasaka T. Promising cell-based therapy for bone regeneration using stem cells from deciduous teeth, dental pulp, and bone marrow. Cell Transplant. 2011;20:1003-13.

24. Wang W, Yuan C, Geng T, Liu Y, Zhu S, Zhang C, et al. EphrinB2 overexpression enhances osteogenic differentiation of dental pulp stem cells partially through ephrinB2-mediated reverse signaling. Stem Cell Res Ther. 2020;11:40.

25. Gu Y, Bai Y, Zhang D. Osteogenic stimulation of human dental pulp stem cells with a novel gelatinhydroxyapatite-tricalcium phosphate scaffold. J Biomed Mater Res Part A. 2018;106:1851-61.

\section{Tables}

Table 1. Sequence information of primers used for quantitative real-time PCR 


\begin{tabular}{|c|c|}
\hline Gene & Primer sequences (forward and reverse, $5^{\prime}-3^{\prime}$ ) \\
\hline GAPDH & GAAGGTGAAGGTCGGAGTCA \\
\cline { 2 - 2 } & GAAGATGGTGATGGGATTTC \\
\hline \multirow{2}{*}{ RUNX2 } & CAGACCAGCAGCACTCCATA \\
\cline { 2 - 2 } ALP & CAGCGTCAACACCATCATTC \\
\cline { 2 - 2 } COLIA1 & ATGAAGGAAAAGCCAAGCAG \\
\cline { 2 - 2 } & ATGGAGACATTCTCTCGTTC \\
\cline { 2 - 2 } Osteocalcin & GTGCTAAAGGTGCCAATGGT \\
\cline { 2 - 2 } & GGCCTCGCTTTCCTTCCTCT \\
\cline { 2 - 2 } & GGCAGCGAGGTAGTGAAGAG \\
\hline
\end{tabular}

Table 2. Initial percentage of dental pulp stem cells (DPSCs) derived from young and elderly patients

\begin{tabular}{|c|c|c|c|c|}
\hline & $\begin{array}{c}\text { Number of } \\
\text { collected cells } \\
\left(\times 10^{4}\right)\end{array}$ & $\begin{array}{c}\text { Number of DPSCs at } \\
70 \% \text { confluence }\left(\times 10^{4}\right)\end{array}$ & $\begin{array}{c}\text { Percentage } \\
\text { of DPSCs } \\
(\%)\end{array}$ & $\begin{array}{c}\text { Average } \\
\text { percentage of } \\
\text { DPSCs (\%) }\end{array}$ \\
\hline (Young) & 1000 & 800 & 80 & \\
Y1 & 1000 & 500 & 50 & 70 \\
Y2 & 2600 & 600 & 71 & \\
Y3 & 670 & 480 & 128 & \\
Y5 & 7 & 9 & & \\
& & & & \\
& & & 54 & \\
(Elderly) & 198 & 107 & 16 & \\
E1 & 60 & 60 & 44 & \\
E3 & 760 & 28 & 90 & \\
E4 & 63 & 90 & & \\
E5 & 100 & & & \\
& & & & \\
& & & & \\
& & & & \\
\end{tabular}

Figures 

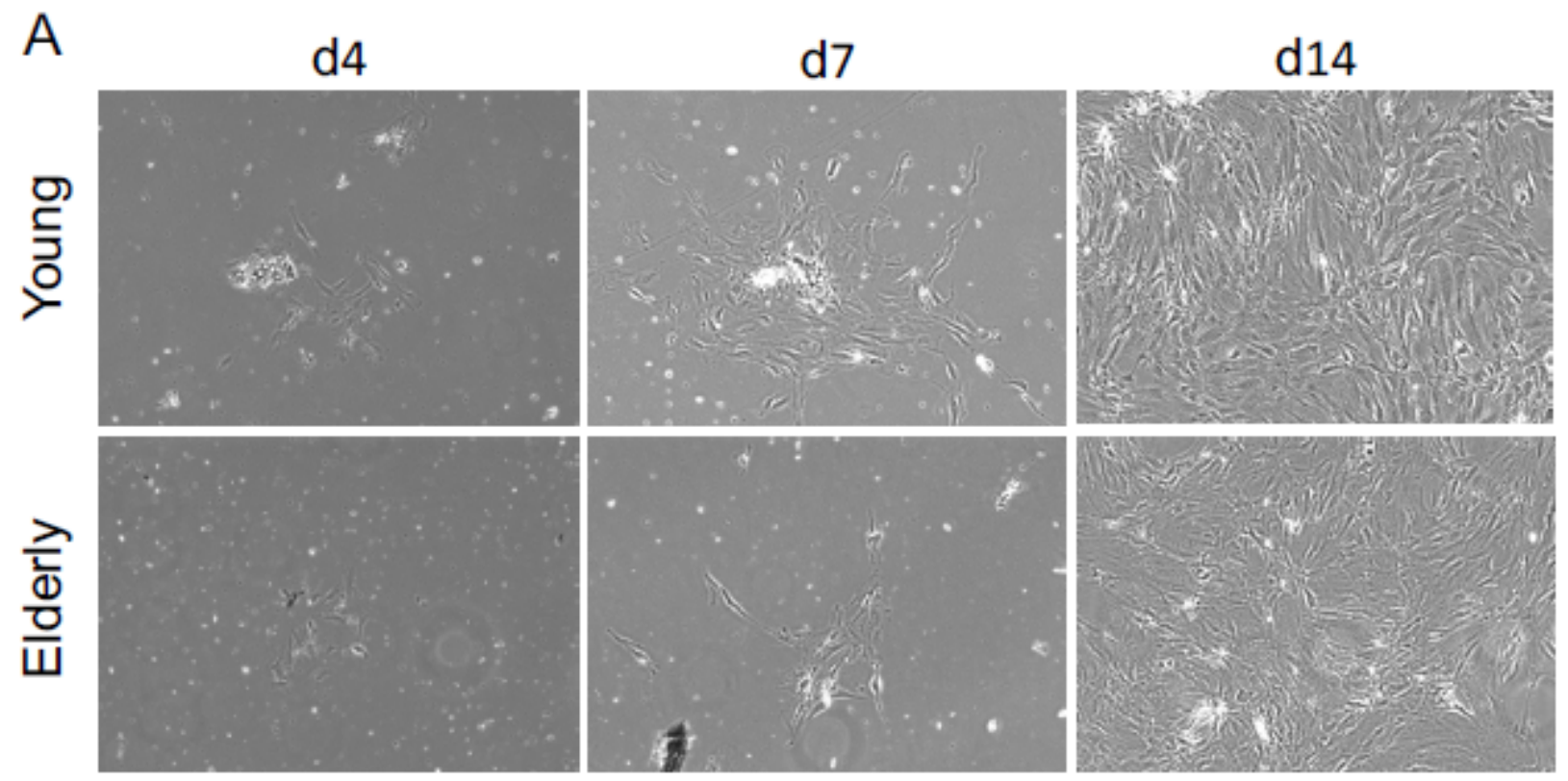

B

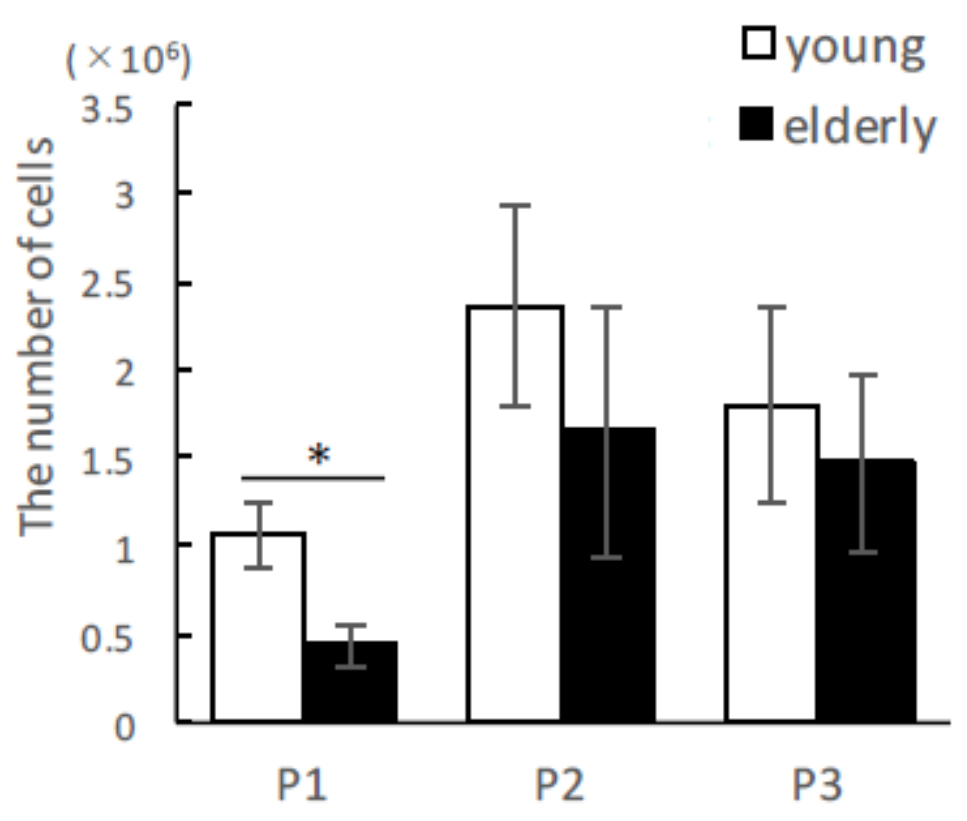

Figure 1

Characterization of dental pulp stem cells (DPSCs) derived from young and elderly patients (A) Cell morphology of DPSCs derived from young and elderly patients at initial culture. Gross view at day 4, day 7, and day 14. (B) Comparison of proliferation potential of DPSCs derived from young and elderly patients in passage 1 (P1) to P3. The number of collected cells was counted at each passage. 


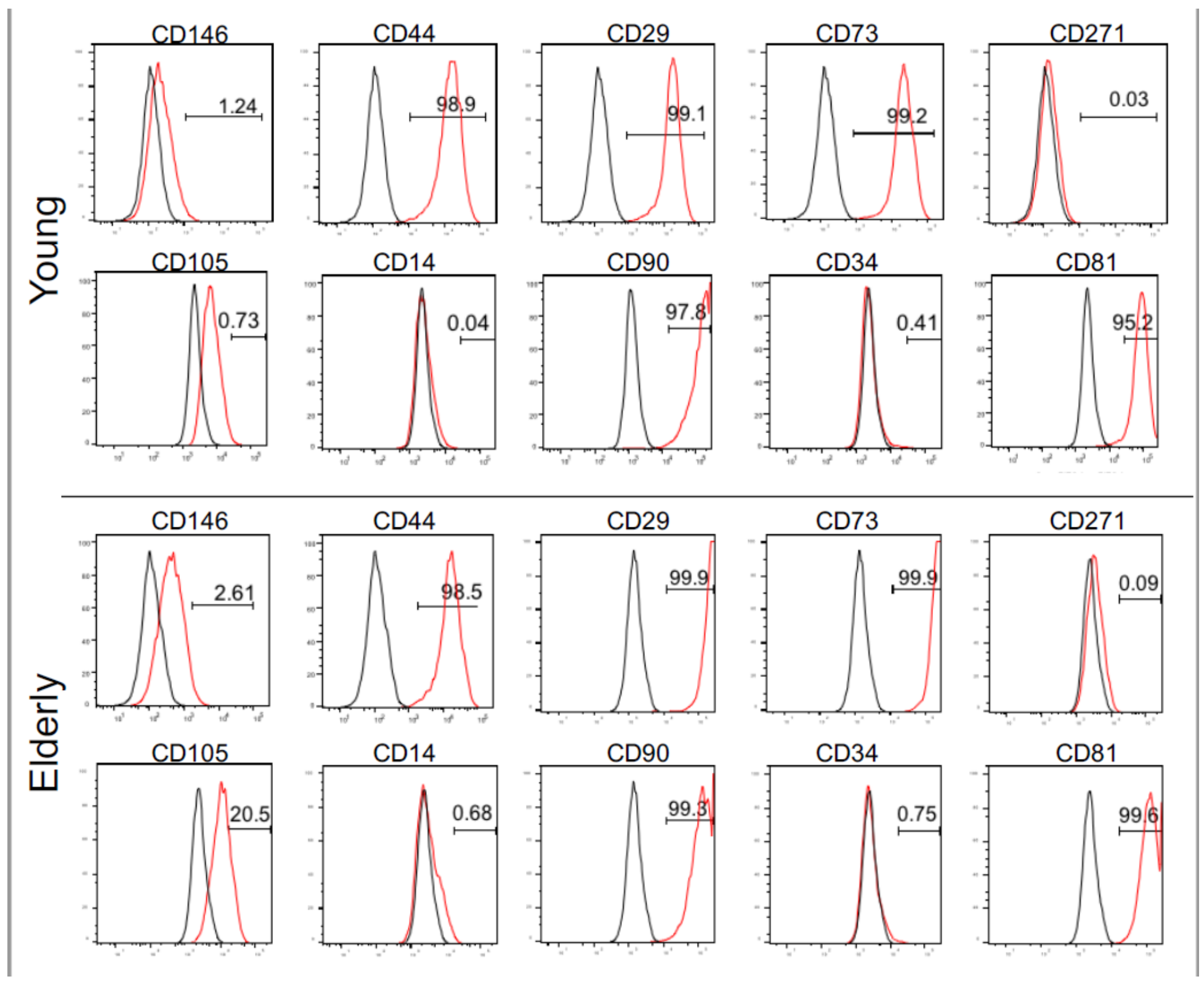

Figure 2

Flow cytometry analysis of dental pulp stem cells (DPSCs) derived from young and elderly patients DPSCs were cultured at passage 3 (P3) or P4 and surface stained with fluorescein isothiocyanateconjugated CD14, CD34, CD44, CD81, CD105, and CD146 and phycoerythrin-conjugated CD29 and CD90. Fluorescent signals were measured using flow cytometry analysis. Control cells are represented by the black curve, and labelled cells are represented by the red curve. 
A
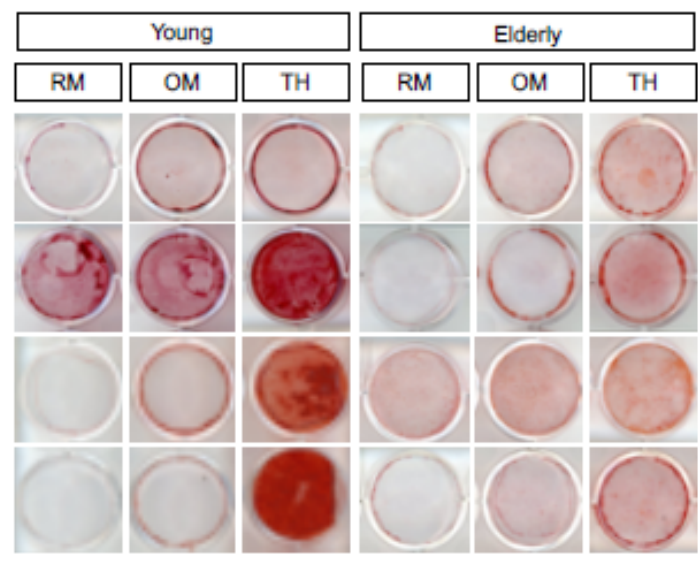

C
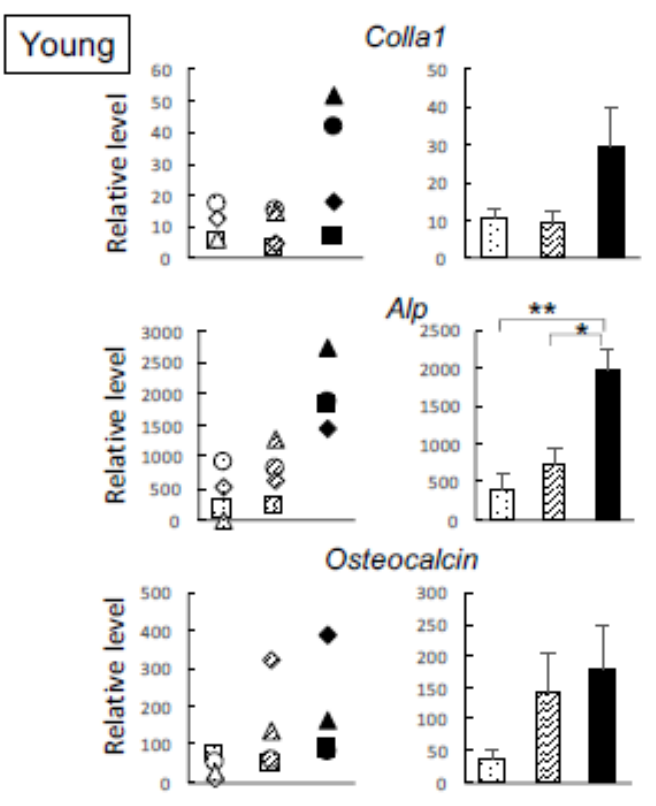

Runx
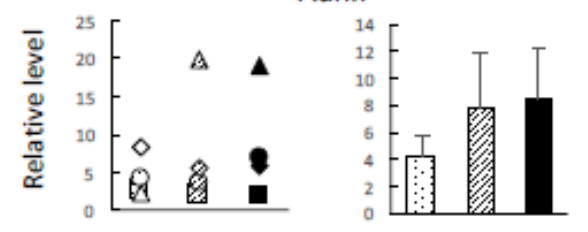

RM

OM

TH

\section{B}

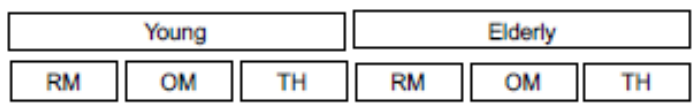
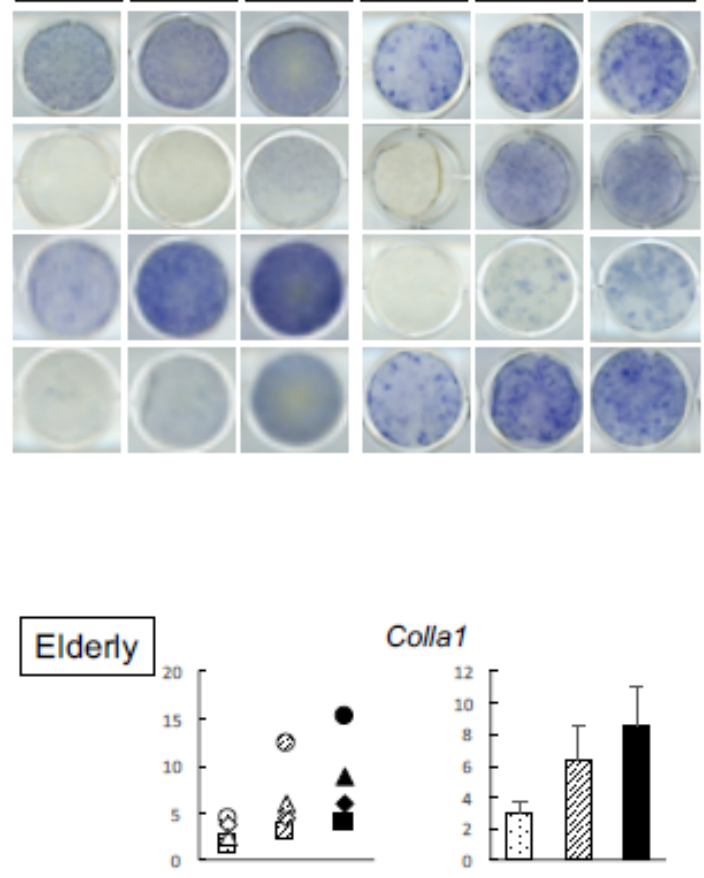

Colla1
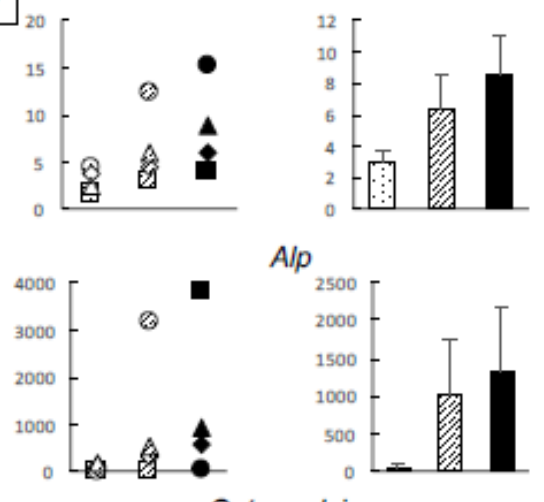

Osteocalcin

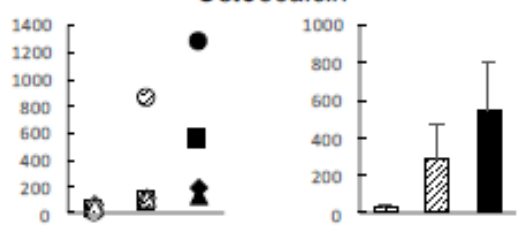

Runx

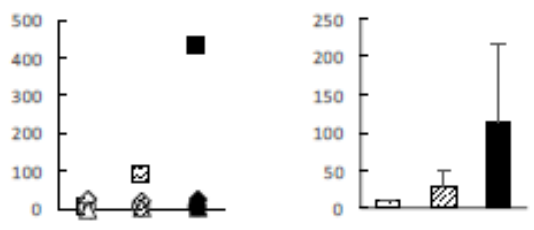

$\begin{array}{ll}\text { RM } & \text { RM } \\ \text { RM } \\ \text { OM } \\ \text { TH } \\ \text { TH }\end{array}$

\section{Figure 3}

Effect of TH on the osteogenic differentiation of dental pulp stem cells (DPSCs) derived from young and elderly patients (A) Young and elderly DPSCs were cultured in regular medium (RM), osteogenic medium $(\mathrm{OM})$, or $\mathrm{OM}$ with $\mathrm{TH}$ for 14 days. Cells were stained with alizarin red $\mathrm{S}$ to detect matrix mineralization ( $\mathrm{n}$ = 4). To detect ALP activity, cells were subjected to ALP staining $(n=4)$. (B) Real-time PCR was performed to determine the expression levels of osteogenic differentiation markers in young and elderly DPSCs 
cultured in RM, OM, or OM with TH for 14 days $(n=4)$. Error bars represent the standard deviation. Statistical analyses were performed using one-way ANOVA $\left({ }^{*} p<0.05,{ }^{\star *} p<0.01\right)$.

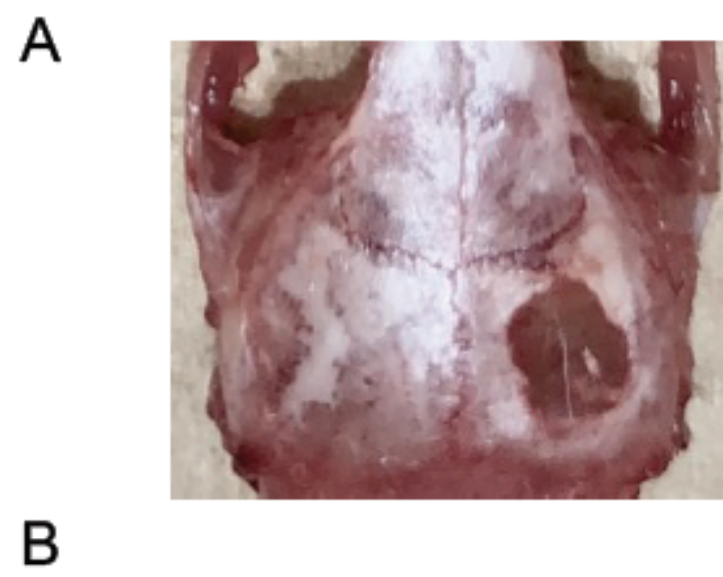

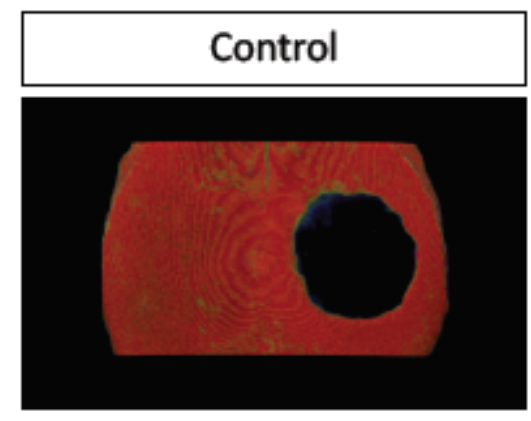
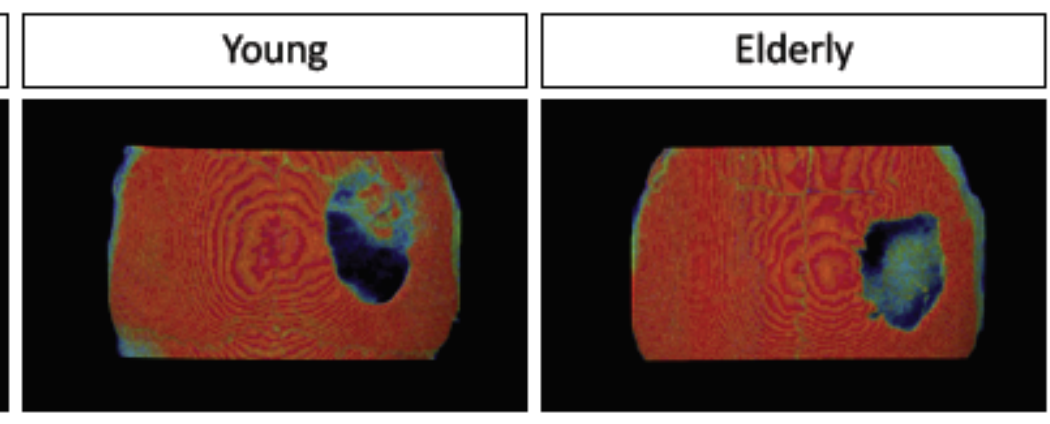

$\mathrm{BMD}(\mathrm{mg} / \mathrm{cm} 3)$

1

\section{C}

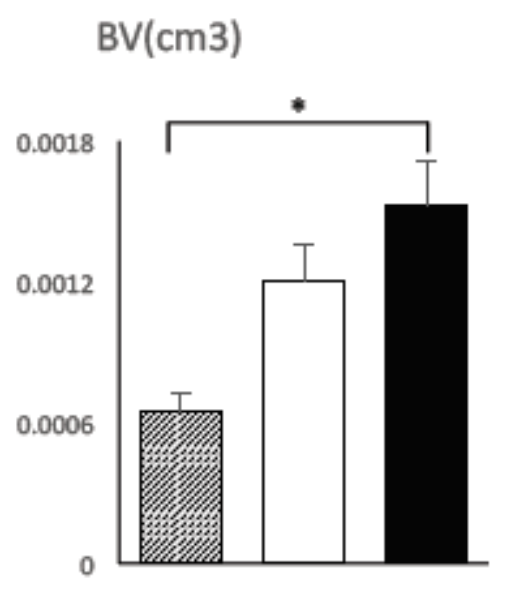

BMC(mg)

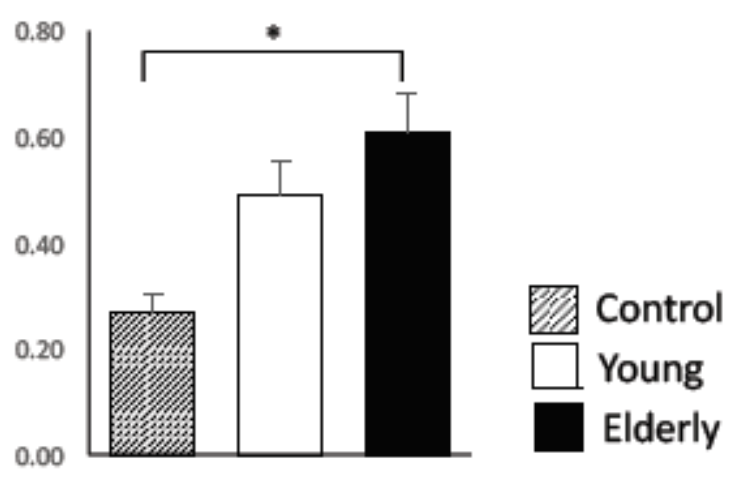

\section{Figure 4}

Radiological findings of mouse calvarial defects 8 weeks after transplantation with cell sheets of THinduced dental pulp stem cells (DPSCs) from young and elderly patients (A) TH-induced DPSCs sheets were cultured on temperature-responsive dishes for 14 days and then transplanted into mouse calvarial defects ( $3.5 \mathrm{~mm}$ in diameter). Eight weeks after transplantation, the calvaria were dissected. (B) Micro- 
computed tomography images indicate the bone mineral density (BMD) values. The experiments were repeated three times, and one representative image is shown. (C) Quantification of bone volume (BV) and bone mineral content (BMC) of a $5 \times 5 \times 3 \mathrm{~mm} 3$ cuboid area in the center of a circular defect. Data are expressed as means \pm standard deviation of five mice per group. ${ }^{*} P<0.05$ by Student unpaired twotailed t-test. 